\title{
A genome-wide map of regulatory elements in zebrafish
}

Yang, H., Luan, Y., Liu, T. et al. Nature https://doi.org/10.1038/s41586-020-2962-9 (2020)

The zebrafish genome was fully sequenced in 2013 and comparison between zebrafish and human genomes revealed that $70 \%$ of human genes have at least one zebrafish orthologue. This genetic similarity makes zebrafish a well-suited model to study human development and disease.

Cis-regulatory elements are DNA regions that control the spatiotemporal transcription of genes and can contribute to human diseases. Although extensive efforts have been made to locate these elements in the mouse and human genome, our knowledge of the cis-regulatory landscape in zebrafish remains limited. In a new study published in Nature, a team of investigators led by Feng Yue from Northwestern University used several state-of-the-art techniques to produce a comprehensive map of cis-regulatory elements, chromatin accessibility and 3D genome organisation in zebrafish. This new resource will be useful for future zebrafish studies aiming at identifying regulatory elements contributing to disease.

The investigators predicted tens of thousands of cis-regulatory elements such as promoters, enhancers and heterochromatin sites across 11 zebrafish adult tissues and 2 embryonic tissues by combining data from assay for transposase-accessible chromatin using sequencing (ATAC-seq) - a technique to assess chromatin accessibility-and chromatin immunoprecipitation with sequencing (ChIP-seq) for a panel of histone modifications. Comparison of results between tissues notably revealed that $71.3 \%$ of identified enhancers were tissue-specific and often located near genes important for tissue-specific functions.

The investigators also used whole-genome bisulfite sequencing (WGBS) to analyse DNA methylation patterns across the genome. They identified unmethylated regions (UMRs) and low-level-methylated regions (LMRs), which overlapped with candidate promoters and enhancers respectively, as well as differentially methylated regions (DMRs) and tissue-specific hypomethylated DMRs (hypoDMRs). Tissue-specific hypoDMRs were enriched with tissue-specific $\mathrm{H} 3 \mathrm{~K} 27 \mathrm{ac}$ histone mark and ATAC-seq signals, suggesting that methylation patterns can identify tissue-specific cis-regulatory elements.

In addition, the investigators performed chromosome conformation capture (Hi-C) to study higher-order chromatin structure in brain and muscle tissues. They predicted thousands of topologically associating domains (TADs) in both tissues, as well as chromatin loops. Hi-C data from the brain was also combined to long-read DNA sequencing to assemble de novo chromosome 4, the rudimentary sex chromosome in zebrafish.

All the sequencing data are deposited in the NCBI Gene Expression Omnibus under accession code GSE134055. All the genomic data can be visualized in the WashU Epigenome Browser (https://epigenome. wustl.edu/zebrafishENCODE/).

\section{Alexandra Le Bras}

Published online: 14 December 2020 https://doi.org/10.1038/s41684-020-00695-7

\section{natureresearch} ACADEMIES

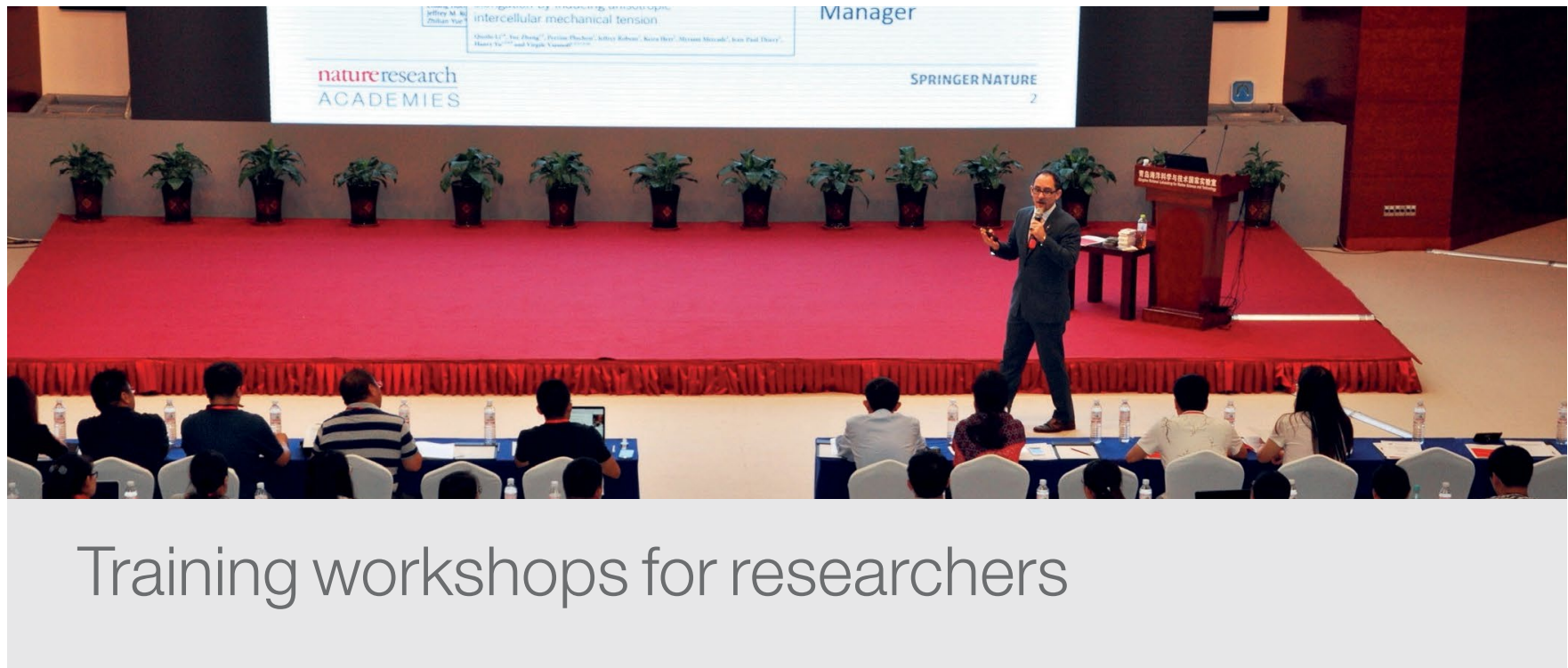

A series of workshops to support researchers, covering topics such as getting published, journal editing, clinical research methodology, and applying for research positions.

Visit partnerships.nature.com/academies to host an academy at your institution. 